\title{
Research on Distributed Energy Transaction based on Blockchain
}

\author{
Christine Sarson \\ University of New South Wales, Australia \\ christine.sarson@cse.unsw.edu.au
}

\begin{abstract}
In recent years, with global warming, environmental pollution, energy resources trend. In the current stage of clean, low-carbon energy development needs, distributed energy, mainly renewable energy such as solar energy, wind energy, and tidal energy, is the main primary energy source for the future energy Internet. Energy blockchain technology is expected to become an important breakthrough to solve the bottleneck of the development of the energy Internet, and its most widely used scenario is distributed energy trading. Distributed energy trading has the characteristics of decentralization, small scale, multiple stakeholders, complex operation status, and diverse control objectives. It coincides with the decentralization, openness, transparency, immutability, and traceability of the blockchain. This article summarizes the application of blockchain technology in distributed energy transactions. First, analyzes the feasibility of the combined application of the two; at the same time, summarizes the application of blockchain technology in distributed energy transactions in different scenarios. And analyze two different blockchain distributed energy transaction modes; secondly, it summarizes the method of increasing the throughput performance of the blockchain network.
\end{abstract}

Keywords: Blockchain, Distributed energy transaction, Transaction mode, Application scenarios

\section{The feasibility of applying blockchain technology in distributed energy transactions}

In the process of development of distributed energy transactions, its control structure has gradually developed from centralized control to completely decentralized. Blockchain has the characteristics of decentralization. It can ensure that all participating nodes in the network have the same status through a consensus algorithm and jointly complete computing tasks. At the same time, distributed energy sources and terminal load points are multi-faceted, power sources are diverse, operating conditions are complex, grid control objectives are diverse, coordinated control is difficult, and controllability is insufficient. Blockchain technology has collective coordination capabilities and uses specific incentive mechanisms to ensure that each block participates in the verification and competition process of data blocks, and promotes its independent coordination and cooperation.

Distributed energy, as an important part of the energy network, realize the interconnection with power generation measurement, power demand side, power trading market, and grid regulation market through two-way communication technology. At the same time, it integrates, optimizes, and dispatches data information at all levels, and participates in power market

Article History:

Received (August 10, 2019), Review Result (September 16, 2019), Accepted (November 14, 2019) 
dispatch. However, distributed energy participants are diverse. Power generation and user-side distributed energy have a small capacity, a large number, and a geographically dispersed position. Related stakeholders are complex. With the opening of policies, market participants are becoming more active, and the demands of different stakeholders are intertwined [1]. The interaction between clusters (microgrids, aggregators, virtual power plants) and between the grid and other nodes is expensive, and the benefit distribution mechanism is not open and transparent to all market participants. Distributed energy and power grids and clusters cannot form a symmetrical two-way choice of information, which increases the cost of credit in the transaction process. Blockchain technology has advantages in transaction applications due to its characteristics. First of all, all nodes in the network jointly participate in the recording of transactions, and the decentralized transaction organization reduces transaction costs. At the same time, as a decentralized distributed ledger, the blockchain stores all the information in the system. All transaction records cannot be tampered with privately and can be traced back, ensuring the safety and reliability of transaction data. Also, the blockchain provides fair information reading permissions for many nodes and can read and write block information at any time. The power grid and users can grasp the changes of schedulable resources during the transaction in time, which is convenient for adjusting the transaction strategy. Therefore, the information in the blockchain is open, transparent, and authentic, ensuring the safety and reliability of transactions.

\section{Application scenarios of distributed energy trading based on blockchain}

\subsection{Virtual power plant power trading}

The virtual power plant activates and utilizes fragmented resources such as a large number of distributed resources, energy storage resources, and community users that are scattered in large numbers, forming a bottom-up management and control structure. Take homes, buildings, communities, parks, etc. as the unit construction, realize interconnection transactions through source-load collaboration and provide auxiliary services to the grid at the same time. However, traditional virtual power plants have problems such as non-open and transparent benefit distribution mechanisms, so the cost of trust required for power transactions is relatively high. The literature proposes a semi-centralized blockchain with the characteristics of both private and public chains and improves the Proof of Work (POW) consensus mechanism of the blockchain to improve the optimal scheduling of virtual power plants. Computational efficiency. The literature introduces the energy blockchain network model into the operation scheduling process of the virtual power plant so that distributed energy can effectively participate in the power market transaction, and with the help of cryptographic characteristics, the virtual power plant can obtain the security of transaction information.

\subsection{Microgrid grid-connected power generation transaction}

A Microgrid is a small power generation and distribution system that combines distributed energy, energy storage equipment, energy conversion equipment, control and protection equipment, and loads through power electronic technology. The advantage is to improve power supply reliability and reduce feeder losses, but there are certain physical limitations. Because the blockchain and the microgrid market have similar distributed topological structures, blockchain technology can be used to simplify the transaction mode, realize point-to-point transactions, promote the nearby consumption of distributed energy, and reduce power transaction costs [2]. The literature designed the overall framework of the microgrid market 
based on the blockchain, made up for the lack of the game model in obtaining global information, and introduced a reward and punishment mechanism to ensure the fairness of individual participation in trading so that the game results tended to optimize the optimal solution. The literature studies microgrid economic dispatch based on the energy blockchain network, and then takes into account energy storage and user-side response, and proposes a multi-time scale microgrid economic dispatch method based on smart contracts to improve the information transparency and data security of the microgrid system and storage security. The literature proposes a direct power transaction mode and strategy between distributed power sources and users in the microgrid based on the blockchain and continuous double auction mechanism and verifies that the method can meet the decentralized, small-scale, and low-cost microgrid power transaction cases. Cost of transaction requirements.

\subsection{Automatic demand response transactions}

In the context of the Energy Internet, as the penetration rate of distributed energy continues to increase, more forms of demand response resources will emerge. Automatic demand response can realize the adjustment of both ends of "source-network-load" to ensure the realtime balance of the power grid. Making full use of the real-time adjustable potential of the load. However, the traditional centralized trading model of the electricity market has been difficult to achieve low-cost, safe, and efficient interaction of users. Blockchain technology can just guarantee the traceability and tamper-proof of transaction information and realize decentralized and credible transactions [3]. Masiello et al [4] proposed a design idea of an integrated information platform based on blockchain protocol standards, centered on information monitoring collection, entry, and statistics, and based on demand response servers, load integrators, and user groups so that the entire system The security is not limited to a central point of failure. Kounelis et al [5] proposed an overall framework for comprehensive demand response resource transactions based on blockchain technology and analyzed the key issues in market transactions under the framework. Tanaka et al [6] takes the demand response transaction of distributed energy as an example to illustrate the detailed process of the transaction, and design and deploy the smart contract of the transaction on the Ethereum private blockchain to verify the effectiveness of the proposed transaction plan. Cutsem et al [7] explored the application method of blockchain smart contracts in demand response bidding transactions and proposed a multi-level demand response management scheme based on blockchain smart contract technical support from the technical level, which can guarantee the support of demand response bidding. Business security transactions.

\subsection{Electric vehicle charging and discharging transactions}

In recent years, in response to the call for energy conservation, emission reduction, and environmental protection, many countries have actively promoted the development of new energy vehicles, and their ownership has gradually increased, but there are problems such as inconsistent charging standards and user privacy leakage [8]. Blockchain technology uses decentralization and trust less methods to realize the secure transaction of digital assets. Chen et al [9] proposed a charging pile sharing scheme based on blockchain technology. The charging alliance chain eliminated the trust barrier between electric vehicle companies and charging pile operators. Decker et al [10] constructed a blockchain-based charging rights transaction mechanism and model. The smart contract executors in the blockchain network fairly distribute the initial charging rights to achieve peer-to-peer digital asset transactions to avoid overloading of power transmission and transformation equipment. 
At present, blockchain technology has preliminary practical applications in the field of distributed energy transactions at home and abroad and has achieved certain results. A typical example of blockchain distributed energy transactions is shown in [Table 1].

Table 1. Typical cases of blockchain distributed energy transactions

\begin{tabular}{|c|c|c|}
\hline Project name & Region & Core Technology \\
\hline $\begin{array}{l}\text { Power Ledger solar } \\
\text { trading }\end{array}$ & Australia & $\begin{array}{l}\text { P2P transactions between producers and consumers who own } \\
\text { distributed energy and other users are realized through smart } \\
\text { contracts, but the internal private chain is used, which has poor } \\
\text { scalability }\end{array}$ \\
\hline $\begin{array}{l}\text { TransActiveGrid } \\
\text { distributed photovoltaic } \\
\text { power sales }\end{array}$ & United States & $\begin{array}{l}\text { Provide a distributed energy trading platform in the Brooklyn } \\
\text { community. Smart meters collect users' electricity consumption } \\
\text { and transaction data and record them on the blockchain, but the } \\
\text { scale of project application is relatively small }\end{array}$ \\
\hline $\begin{array}{l}\text { Share \& Charge Electric } \\
\text { Vehicle Charging } \\
\text { Platform }\end{array}$ & Germany & $\begin{array}{l}\text { Platform users look for charging piles on the client-side and } \\
\text { use smart contracts to achieve transaction settlement between } \\
\text { users and charging pile operators, but the tokens issued by the } \\
\text { platform are not promotional }\end{array}$ \\
\hline $\begin{array}{l}\text { BittWatt blockchain } \\
\text { project }\end{array}$ & $\begin{array}{l}\text { United } \\
\text { Kingdom }\end{array}$ & $\begin{array}{c}\text { Establish an intelligent demand response platform based on } \\
\text { Ethereum, where generators and consumers can match } \\
\text { transactions through smart contracts }\end{array}$ \\
\hline Fortum Energy System & Finland & $\begin{array}{l}\text { Build a demand response platform based on the blockchain, } \\
\text { with the help of smart contracts to enable the subject to } \\
\text { automatically respond to the compensation demand proposed } \\
\text { by the system }\end{array}$ \\
\hline $\begin{array}{l}\text { Scanergy blockchain } \\
\text { project }\end{array}$ & $\begin{array}{l}\text { European } \\
\text { Union }\end{array}$ & $\begin{array}{l}\text { Based on the smart contract of Ethereum to realize P2P direct } \\
\text { transaction, the smart contract generates corresponding virtual } \\
\text { currency and provides it to the green power producer, but the } \\
\text { economic model, transaction security, data privacy, and other } \\
\text { aspects of the project need to be improved }\end{array}$ \\
\hline $\begin{array}{l}\text { Sun Exchange Solar } \\
\text { Equipment Crowdfunding } \\
\text { Project }\end{array}$ & Africa & $\begin{array}{l}\text { Raise external funds with the help of the blockchain platform } \\
\text { to help African countries build photovoltaic projects, but the } \\
\text { project has relatively little correlation with energy distributed } \\
\text { trading, optimized operation, etc. }\end{array}$ \\
\hline $\begin{array}{c}\text { De La Salle University } \\
\text { Project }\end{array}$ & Philippines & $\begin{array}{l}\text { The project is based on the campus microgrid project at De La } \\
\text { Salle University. The decentralized characteristics of the } \\
\text { blockchain can support users in different buildings to conduct } \\
\text { P2P power direct transactions, but the project is small in scale } \\
\text { and the experimental stage }\end{array}$ \\
\hline $\begin{array}{l}\text { WePower Green Energy } \\
\text { Trading Platform }\end{array}$ & Gibraltar & $\begin{array}{l}\text { Build a blockchain platform, issue energy tokens to raise } \\
\text { funds, and promote the development of global renewable } \\
\text { energy }\end{array}$ \\
\hline Smart microgrid project & Israel & $\begin{array}{c}\text { A microgrid pilot project based on blockchain technology and } \\
\text { artificial intelligence has been built to achieve green power } \\
\text { certification, power trading, regional energy optimization } \\
\text { management, and P2P functions }\end{array}$ \\
\hline $\begin{array}{l}\text { Shekou Energy } \\
\text { Blockchain Project }\end{array}$ & China & $\begin{array}{c}\text { Users can select the type of electric energy through the trading } \\
\text { platform to realize the P2P transaction of renewable energy } \\
\text { between the user and the power station, but the reward } \\
\text { mechanism needs to be improved }\end{array}$ \\
\hline $\begin{array}{l}\text { Zhejiang Electric Power } \\
\text { Marketing Contract } \\
\text { Management Application } \\
\text { Project }\end{array}$ & China & $\begin{array}{l}\text { Using blockchain technology to ensure the consistency of the } \\
\text { electronic contract data information stored by the various } \\
\text { stakeholders in the power market, but the project has a } \\
\text { relatively single application scenario for the blockchain }\end{array}$ \\
\hline $\begin{array}{l}\text { TenneT Home Energy } \\
\text { Storage Project }\end{array}$ & Europe & $\begin{array}{l}\text { Combine home storage batteries with blockchain to perform } \\
\text { real-time charge and discharge management to alleviate } \\
\text { congestion, but the degree of decentralization is low }\end{array}$ \\
\hline
\end{tabular}




\section{Distributed energy trading model based on blockchain}

Blockchain-based distributed energy transaction settlement models mainly include P2P transactions and centralized clearing. There are differences in the transaction process between the two modes. [Table 2] shows the comparison between P2P transactions and the centralized clearing transaction process. The most significant difference between the two is that in P2P transactions, users with electricity trading qualifications and distributed energy generators have completed transaction matching at the application layer through the website or DAPP in Phase 1 , without the need for matching in the blockchain. In the centralized clearing mode, the block link in stage 3 will match the buyers and sellers after receiving the quotations from the buyers and sellers.

\subsection{P2P transaction}

Blockchain-based P2P transactions take advantage of the decentralization and traceability characteristics of the blockchain. Based on the P2P structure of the blockchain, when conducting electricity transactions, there are only buyers and sellers, and there is no central node [10]. Before the transaction, the buyer and the seller agree on the content of the contract. During the transaction, the smart contract is automatically executed according to the quotation of both parties, and the buyer and the seller do not need to negotiate a price. King [11] proposed a photovoltaic transaction mechanism based on blockchain incentives, which can encourage free, flexible, and single transaction settlement among users. Under the trading mechanism using reputation value, users can obtain more satisfactory transactions. Hirose et al [12] proposed a demand response framework for decentralized cooperation. Through the complete decentralization of blockchain technology, a trusted communication medium can be established between participants, and autonomous monitoring and billing can be implemented through smart contracts. Without relying on centralized aggregators or utilities. Kokoris-Kogias et al [13] established a competition game model for market entities based on blockchain. In this model, the information of energy storage entities does not need to be fed back to the control center, but point-to-point information interaction and asset transfer with other entities. Shalini et al [14] proposes a distributed energy trading model based on contract orders, and users' matching transactions are all realized through contract orders.

Table 2. Comparison of $\mathrm{P} 2 \mathrm{P}$ transaction and centralized clearing transaction process

\begin{tabular}{|c|c|c|c|}
\hline Transaction mode & Stage 1 & Stage 2 & Stage 3 \\
\hline P2P transaction & $\begin{array}{c}\text { Request for purchase and sale } \\
\text { of electricity to request } \\
\text { transaction matching between } \\
\text { buyers and sellers }\end{array}$ & $\begin{array}{c}\text { Information transfer } \\
\text { to the blockchain }\end{array}$ & $\begin{array}{c}\text { Transaction clearing stores } \\
\text { transaction information }\end{array}$ \\
\hline $\begin{array}{c}\text { Centralized } \\
\text { clearing }\end{array}$ & $\begin{array}{c}\text { Request for purchase and sale } \\
\text { of electricity }\end{array}$ & $\begin{array}{c}\text { Information transfer } \\
\text { to the blockchain }\end{array}$ & $\begin{array}{c}\text { Buyer and seller transaction } \\
\text { matching transaction clearing } \\
\text { storage transaction information }\end{array}$ \\
\hline
\end{tabular}

\subsection{Centralized clearing}

The centralized clearing model mainly refers to the use of the decentralized characteristics of the blockchain to gather the quotations and needs of market participants for matching, forming a supply-demand curve, and then clearing. According to different quotation methods, it is mainly divided into collections Bidding and continuous bilateral auctions. 
In the in-call auction, after buyers and sellers give quotations, the blockchain platform selects according to the quotations and demand of the buyers and sellers to reach the transaction. Wu et al [15] used the Vickrey Clarke Groves (VCG) auction rules to motivate producers and consumers to make rational quotations. The quotations of users and distributed energy were submitted to the blockchain platform in a confidential state before the start of the trading cycle. When the quotation information is made public, all valid quotations will be entered into the clearing queue from low to high, until the deviation power balance constraint is met. The Underwood [14] encodes the bidding rules into smart contracts by the demander of electricity and the generator of electricity, and it is simulated and implemented on the Ethereum blockchain platform. Although the transaction efficiency of call auction is higher than that of $\mathrm{CAD}$ auction, and it eliminates the game cost of producers and consumers, the quotations given by buyers and sellers cannot be modified, so it is difficult to coordinate to achieve the optimal, and the participants may not obtain the maximum benefit [16].

Continuous bilateral auction refers to the situation where buyers and sellers exist in manyto-many forms, market participants can submit quotations at any time, and the quotations are submitted to the auction center to be responsible for matching. Buyers and sellers are sorted and matched according to the principle of "price first, time first". To illustrate the above auction mechanism, the auction process of each node is illustrated with examples. Suppose the user node is the buyer, and define the vector $A=\left[A_{1}^{t_{1}}, A_{2}^{t_{2}}, A_{3}^{t_{3}}, A_{4}^{t_{4}}\right]$, , where Atik indicates that user $\mathrm{k}$ has completed the purchase price of electricity at time $t_{i}$, The distributed energy service provider node is the seller, and the definition vector is $B=\left[B_{1}^{t_{1}}, B_{2}^{t_{2}}, B_{3}^{t_{3}}, B_{4}^{t_{4}}\right]$, , where Btij represents the distributed energy service provider $\mathrm{j}$ in $t_{i}$ complete the electricity sales quotation at any time, here assume $t_{1}<t_{2}<t_{3}<t_{4}$. The quotation information of buyers and sellers is shown in [Table 3], and the order of quotations and transaction matching are shown in [Table 4]. Buyers are sorted in descending order of bids, and sellers are sorted in ascending order of selling prices. Matching occurs when the selling price is higher than the selling price, and the selling price is the average price of the two. In the Ramya et al [17], buyers and sellers first complete transaction matching through a continuous two-way auction mechanism, and propose an adaptive and aggressive trading strategy, so that the transaction parties can adjust their quotations in time according to market changes. Nikolic et al [18] Twoway auction and $\mathrm{P} 2 \mathrm{P}$ trading mechanisms introduce the charging rights trading market. For the part where the buyer's price is higher than the seller's price, the two-way auction market is used to match the transaction; the part where the buyer's price is lower than the seller's price is through the P2P trading market Listing transactions to maximize the optimal allocation of charging resources.

Table 3. Quotation information of buyers and sellers

\begin{tabular}{|c|c|c|c|}
\hline Buyer & Bid & Seller & Selling price \\
\hline$A_{1}^{t_{1}}$ & 80.00 & $B_{1}^{t_{1}}$ & 84.00 \\
\hline$A_{2}^{t_{2}}$ & 86.00 & $B_{2}^{t_{2}}$ & 80.00 \\
\hline$A_{3}^{t_{3}}$ & 82.00 & $B_{3}^{t_{3}}$ & 84.00 \\
\hline$A_{4}^{t_{4}}$ & 80.00 & $B_{4}^{t_{4}}$ & 81.00 \\
\hline
\end{tabular}


Table 4. Quotation order and transaction matching

\begin{tabular}{|c|c|c|c|c|c|}
\hline Buyer descending & Bid & Seller ascending & Selling price & Does it match & Final price \\
\hline$A_{1}^{t_{1}}$ & 80.00 & $B_{2}^{t_{2}}$ & 80.00 & $\mathrm{Y}$ & 80.00 \\
\hline$A_{4}^{t_{4}}$ & 80.00 & $B_{4}^{t_{4}}$ & 81.00 & $\mathrm{Y}$ & 80.50 \\
\hline$A_{3}^{t_{3}}$ & 82.00 & $B_{1}^{t_{1}}$ & 84.00 & $\mathrm{Y}$ & 83.00 \\
\hline$A_{2}^{t_{2}}$ & 86.00 & $B_{1}^{t_{1}}$ & 84.00 & $\mathrm{~N}$ & - \\
\hline
\end{tabular}

\section{Blockchain technology to improve transaction network throughput performance}

Blockchain technology inherently exists in the trilemma, that is, it cannot have the three characteristics of decentralization, security, and efficient operation at the same time. As the number of participating nodes and running time increase, the data storage system of the blockchain will face greater capacity and maintenance pressure. This will make the throughput of transactions lower and limit the speed of transactions. To solve these problems, developers mainly improve the processing performance of the blockchain from several aspects such as the design of the consensus algorithm, the expansion of the chain, and the cross-chain technology.

\subsection{Improvement of the consensus mechanism}

The consensus mechanism is the core issue of blockchain technology in a decentralized environment. At present, most of the theoretical assumptions of the electricity trading market are based on the operation model of the public chain and the POW consensus mechanism. The world's most famous public chain projects are Bitcoin, Ethereum, and Enterprise Operating System (EOS), so you can see the status quo of public chains by studying them.

Bitcoin adopts the POW consensus mechanism. With the emergence of more powerful ASIC mining machines, the computing power of the entire network is almost monopolized by several major mining pools, so the decentralization of Bitcoin has declined. Bitcoin's system throughput (TPS) is only about 7 transactions per second, which is completely unsuitable for daily high-frequency, small-value transfers. But Bitcoin is the best in terms of security.

Ethereum also uses the POW consensus mechanism, but to solve the problem of computing power monopoly, the Proof of Stake (POS) consensus mechanism will be adopted in the future. Ethereum's TPS is slightly higher than Bitcoin, 7-15 transactions per second, but because Ethereum is a smart contract platform, its application scenarios are more complicated and more prone to congestion than Bitcoin. In addition, Ethereum is second only to Bitcoin in terms of security [70].

In the first edition of the EOS white paper, the consensus mechanism of Proof of Share Authorization (DPOS) was adopted. Now it is changed to Byzantine Fault Tolerance (BFT)Byzantine Fault Tolerant Equity Delegated Consensus Mechanism (DPOS). Its TPS is the highest among the three public chains, reaching 3000 4000 pens. However, the EOS network has only 21 nodes, so it is also the worst of the three in terms of security. 
Table 5. Comparison of characteristics of 5 typical consensus mechanisms

\begin{tabular}{|c|c|c|c|c|c|}
\hline Characteristic & POW & POS & DPOS & PBFT & RBFT \\
\hline $\begin{array}{c}\text { Energy } \\
\text { consumption }\end{array}$ & High & Low & Low & Low & Low \\
\hline $\begin{array}{c}\text { Node } \\
\text { management }\end{array}$ & Public & Public & Public & $\begin{array}{c}\text { Accurate } \\
\text { mechanism }\end{array}$ & $\begin{array}{c}\text { Access } \\
\text { mechanism }\end{array}$ \\
\hline $\begin{array}{l}\text { Block } \\
\text { production } \\
\text { speed }\end{array}$ & $\begin{array}{l}\text { Slow speed, } \\
\text { unable to meet the } \\
\text { needs of high- } \\
\text { frequency small } \\
\text { transactions }\end{array}$ & Faster & $\begin{array}{l}\text { Reaching } \\
\text { second-level } \\
\text { consensus } \\
\text { verification }\end{array}$ & $\begin{array}{l}\text { Slow speed, } \\
\text { suitable for } \\
\text { private chain or } \\
\text { consortium } \\
\text { chain }\end{array}$ & $\begin{array}{c}\text { Fast speed, } \\
\text { TPS } \geq 104\end{array}$ \\
\hline Data throughput & Low & High & High & High & High \\
\hline safety & $\begin{array}{l}\text { Higher can only } \\
\text { withstand less } \\
\text { than } 50 \% \text { of the } \\
\text { hash rate attack }\end{array}$ & $\begin{array}{c}\text { High, can } \\
\text { only } \\
\text { withstand less } \\
\text { than } 50 \% \\
\text { equity attacks }\end{array}$ & $\begin{array}{l}\text { High, can only } \\
\text { withstand less } \\
\text { than } 50 \% \text { of } \\
\text { verification } \\
\text { attacks }\end{array}$ & $\begin{array}{l}\text { Low, can only } \\
\text { withstand no } \\
\text { more than } 1 / 3 \\
\text { of the nodes to } \\
\text { do evil }\end{array}$ & $\begin{array}{l}\text { Low, can } \\
\text { only } \\
\text { withstand } \\
\text { no more } \\
\text { than } 1 / 3 \text { of } \\
\text { the nodes to } \\
\text { do evil }\end{array}$ \\
\hline $\begin{array}{l}\text { Is there a } \\
\text { professional } \\
\text { bookkeeper }\end{array}$ & No & No & Yes & Yes & Yes \\
\hline Scalability & Great & Great & Great & Difference & Difference \\
\hline
\end{tabular}

To shorten the time to reach a consensus on distributed energy transactions on the blockchain, there are currently two main ways to grant nodes different permissions and change the consensus mechanism. Tanaka et al [6] uses an improved indirect verification method to verify the workload proof, that is, a certain number of third-party nodes are used for verification, which can not only improve the computational efficiency but also effectively avoid the Byzantine Generals problem.

Franklin et al [19] based on credit-based rewards and punishments and voting results to choose and optimize two core solutions, and proposed an improved DPOS consensus mechanism, which can significantly reduce the probability of abnormal nodes acquiring the identity of proxy nodes and shorten the time for abnormal proxy nodes to be kicked out of the proxy. Thereby weakening the influence of abnormal nodes on the system and enhancing the security of the system. In a weakly centralized blockchain, the number of light nodes is more than the number of full nodes. The verification form of the MPT (Merkle Patricia Tree) tree is adopted, and the path of the tree is stored in an encoding method. Seung-Min et al [20] uses the consensus mechanism of MPT, the transaction Merkel tree retains all the information of the transaction, and the state Merkel tree retains the state changes of the transaction. If a new node is added to the block, only the leaf nodes of the state tree need to be added. At present, the consensus mechanism of the new research includes the sharding algorithm and the combination of PBFT and POS consensus to form a new Proof of Stake Velocity (POSV) consensus mechanism, etc.

\subsection{Off-chain expansion}

The improvement to the consensus algorithm is called Layer 1 on-chain extension, but it is very time-consuming to record every transaction on the blockchain network into the blockchain. Now a new Layer 2 solution is proposed, called off-chain extension. The off-chain expansion technology mainly includes side-chain assistance, multi-chain fusion, and state channels. The side chain is to separate a chain from the public chain, and return to the main chain after a series of transactions are completed, to achieve the purpose of protecting privacy 
by isolating blockchain data. The solution of transaction matching or distributed optimization algorithm can be placed under the chain, and the main chain is only responsible for the arbitration or recording of transaction results [21]. The Lightning Network transfers transactions to off-chain processing without the need for consensus confirmation on the main chain, thereby improving transaction efficiency. Ethereum Plasma uses POS off-chain, and users can collectively run DAPP through a peer-to-peer network [22]. When multi-chain integration, transaction nodes in the blockchain network can establish their private chain or sub-chain, and place transaction matching in the private chain, and store data with higher security requirements in the main chain. Zeinab et al [23] designed a new hybrid blockchain storage mode, combining the security of public chains with the efficiency of private chains, and using the efficiency of private chains to solve the inefficiency of the initial blockchain. At the same time, it inherits the security and immutability of the initial blockchain. Tesnim et al [24] designed a blockchain with the characteristics of both public and private chains, which not only effectively controls energy grid connection, but also uses a decentralized platform to ensure fair participation of all subjects. The channel is on the private blockchain, and both parties of the communication have all the information, and each communication needs to be verified and signed.

\section{Future development of distributed energy trading based on blockchain}

\subsection{Development challenges}

With the development of energy blockchain, full nodes in the network need to store more transaction data, requiring blocks to have enough storage space. Blockchain network communication will also face delay problems, which will lead to bifurcation. And the current underlying blockchain network cannot support the development of higher systems.

Blockchain technology guarantees the automatic execution of smart contracts and facilitates the smooth progress of distributed energy transactions. If there is a coding error in the contract, it is usually difficult to perform a rollback or fork. Smart contracts will also have problems such as missing responsible parties, timestamp attacks, and re-entry vulnerabilities. In distributed energy transactions, the participant is a virtual account, and the issue of accountability is likely to result in the lack of a responsible entity [25]. And some malicious nodes will use the timestamp in the contract as a trigger condition to obtain improper income. In addition, when one contract calls another contract, the current contract will be rolled back and suspended, which will cause the contract to be called multiple times, causing security issues.

Security and privacy have always been the core issue of blockchain technology. It mainly includes double-spending attacks and mining pool attacks. A double-spend attack refers to spending a single amount of money multiple times, because there will be a delay in the consensus process of network nodes, and malicious nodes will use a single amount of money to trade with multiple nodes at the same time. A mining pool attack is a classic blockchain attack. A malicious mining pool deliberately hides new blocks without publishing them and continues to mine on the hidden blockchain, causing the blockchain to fork.

To deploy distributed energy transactions between users to the actual grid operator, the physical model of the grid operator cannot be constructed, but the blockchain is difficult to integrate well with the complex physical model. In addition, the integration of local distributed energy into the power grid should take into account the user building model, which can take advantage of the flexibility provided by various entities commonly found in buildings. However, there is currently no innovative general framework that can be used in local 
distributed energy production. Manage energy in a flexible smart building community. Therefore, to realize distributed energy trading, we must continue to explore distributed clearing methods that take into account the constraints of building models and physical topology.

\subsection{Development prospects}

At present, the carbon emission market has the problem of a large workload of carbon emission right certification, and the issuance and tracking of green certificates are difficult. With the help of the non-tamperable, traceable, open, and transparent features of the blockchain, a safe and reliable distributed trading platform can be established. The platform can simplify the carbon emission right certification process, effectively avoid fraud in carbon emissions; reduce the certification cost of green certificates, and improve the liquidity of certificates.

\section{Conclusion}

This article systematically summarizes and summarizes the application of blockchain technology in distributed energy transactions. Blockchain technology not only realizes distributed energy $\mathrm{P} 2 \mathrm{P}$ transactions but also ensures centralized and effective supervision. With the increase of distributed energy transaction nodes, the throughput performance of the blockchain is bound to decline. The design of a practical consensus mechanism and off-chain expansion processing technology can improve the efficiency of network transactions. On this basis, the two transaction modes and four typical scenarios of the energy blockchain are analyzed and summarized. Finally, the problems that need to be solved urgently in the future development of blockchain distributed energy trading are put forward, and its development prospects are pointed out. This article is expected to provide a technical reference for the theoretical research and engineering practice demonstration of blockchain distributed energy transactions.

\section{References}

[1] N. Z. Aitzhan and D. Svetinovic, "Security and privacy in decentralized energy trading through multi signatures, blockchain and anonymous messaging streams," IEEE Transactions on Dependable and Secure Computing, vol.15, no.5, pp.840-852, (2018)

[2] L. Xue, Y. Teng, and Z. Zhang, "Blockchain technology for the electricity market in a microgrid," IEEE 2017 $2^{\text {nd }}$ International Conference on Power and Renewable Energy (ICPRE), pp.704-708, (2017)

[3] X. Wu, B. Duan, and Y. Yan, "M2M blockchain: the case of demand-side management of the smart grid," IEEE $23^{\text {rd }}$ International Conference on Parallel and Distributed Systems (ICPADS), pp.810-813, (2017)

[4] R. Masiello and J. R. Aguero, "Sharing the ride of power," IEEE Power and Energy Magazine, vol.14, no.3, pp.70-78, (2016)

[5] I. Kounelis, G. Steri, and R. Giuliani, "Fostering consumers' energy market through smart contracts," IEEE International Conference in Energy and Sustainability in Small Developing Economies (ES2DE), (2017)

[6] K. Tanaka, K. Nagakubo, and R. Abe, "Blockchain-based electricity trading with digital grid router," IEEE International Conference on Consumer Electronics, (2017)

[7] O. V. Cutsem, D. H. Dac, and P. Boudou, "Cooperative energy management of a community of smart-buildings: A blockchain approach,” International Journal of Electrical Power and Energy Systems, pp.117, (2019)

[8] L. Thomas, C. Long, and P. Burnap, "Automation of the supplier role in the GB power system using blockchainbased smart contracts,” CIRED-Open Access Proceedings Journal, no.1, pp.2619-2623, (2017) 
[9] S. Chen and C. C. Liu, "From demand response to transactive energy: State of the art," Journal of Modern Power Systems and Clean Energy, vol.5, no.1, pp.1-10, (2017)

[10] C. Decker and R. Wattenhofer, "Information propagation in the bitcoin network," $13^{\text {th }}$ IEEE International Conference on Peer-to-Peer Computing(P2P), (2013)

[11] S. King and S. Nadal, "PPcoin: Peer-to-peer cryptocurrency with proof-of-stake," Self-published paper, pp.19, (2012)

[12] S. Hirose and H. Kuwakado, "Redactable signature scheme for tree-structured data based on Merkle tree," International Conference on Security and Cryptography (SECRYPT), pp.1-8, (2015)

[13] E. Kokoris-Kogias, P. Jovanovic, and L. Gasser, "OmniL edger: A secure, scale-out, decentralized ledger via sharing," IEEE Symposium on Security and Privacy, pp.583-598, (2018)

[14] S. Shalini and H. Santhi, "A survey on various attacks in bitcoin and cryptocurrency," IEEE International Conference on Communication and Signal Processing (ICCSP), pp.0220-0224, (2019)

[15] L. Wu, K. Meng, S. Xu, "Democratic centralism: A hybrid blockchain architecture and its applications in energy internet," IEEE International Conference on Energy Internet, (2017)

[16] S. Underwood, "Blockchain Beyond Bitcoin," Communications of the ACM, vol.59, no.11, pp.15-17, (2016)

[17] R. Ramya and T. Sasikala, "An efficient Minkowski distance-based matching with Merkle hash tree authentication for biometric recognition in cloud computing," Soft Computing, vol.23, no.24, pp.15, (2019)

[18] I. Nikolic, A. Kolluri, and I. Sergey, "Finding the greedy, prodigal, and suicidal contracts at scale," Proceedings of the 34th Annual Computer Security Applications Conference, New York, pp.653-663, (2018)

[19] S. Franklin, E. Susan, and D. Sophia, "Writing safe smart contracts in flint," Conference Companion of the 2nd International Conference on Art, Science, and Engineering of Programming, Nice, pp.218-219, (2018)

[20] L. Seung-Min, P. Soojin, and B. Young, "Formal specification technique in smart contract verification," 2019 International Conference on Platform Technology and Service (PlatCon), Jeju, pp.1-4, (2019)

[21] M. Yvonne and A. David, "Survey of formal verification methods for smart contracts on the blockchain," 2019 10th IFIP International Conference on New Technologies, Mobility and Security, Canary Islands, pp.1-6, (2019)

[22] S. Amani, B. Myriam, and M. Bortin, "Towards verifying Ethereum smart contract bytecode in Isabelle HOL," Proceedings of the 7th ACM SIGPLAN International Conference on Certified Programs and Proofs, Los Angeles, pp.66-67, (2018)

[23] N. Zeinab, Y. P. Pierre, and D. Frederic, "Model-checking of smart contracts," The 2018 IEEE International Conference on Blockchain, Halifax, pp.980-987, (2018)

[24] A. Tesnim and B. Kei-Leo, "Formal verification of smart contracts based on user and blockchain behaviors models," 2018 9th IFIP International Conference on New Technologies, Mobility and Security (NTMS), Paris, pp.1-5, (2018) 
Research on Distributed Energy Transaction based on Blockchain

This page is empty by intention. 